\title{
Convivencia escolar en Latinoamérica: Una revisión bibliográfica
}

\author{
School Coexistence in Latin America: A Literature Review
}

\section{Convivência escolar na América Latina: uma revisão bibliográfica}

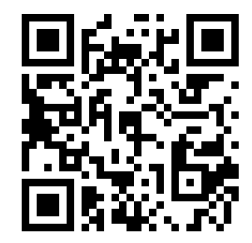

\author{
Jonathan Andrades-Moya \\ Universidad Católica del Maule \\ Talca, Chile \\ jandradesmoya@gmail.com \\ https://orcid.org/0000-0001-5371-9412
}

Recibido • Received • Recebido: 28 / 09 / 2018

Corregido • Revised • Revisado: 04/ 03 / 2020

Aceptado • Accepted • Aprovado: 01 / 04 / 2020

Resumen: La convivencia escolar ha sido investigada en áreas que van desde la violencia escolar, hasta la inclusión educativa. Esto la ha transformado en un tema que no suele investigarse como objeto principal, sino más bien como una variable que se asocia a otros factores, tales como: problemas con los resultados de aprendizaje, conflictos, malas prácticas docentes, inclusión, violencia escolar. Por este motivo se planteó el objetivo de analizar la producción académica presente en revistas Web o Science, Proquest, Scielo y Scopus, de Latinoamérica desde el 2010 hasta junio del 2018, en torno a las problemáticas asociadas a la convivencia escolar. Para su desarrollo se optó por la revisión bibliográfica de tipo descriptiva, utilizando 38 artículos de diversos países. Dentro de los principales resultados se resalta que el tema más investigado es aquel relacionado con los factores (antes mencionados) que influyen en la convivencia escolar. Por último, cabe anotar, que una de las características más destacadas es la asociación entre convivencia escolar y violencia escolar.

Palabras claves: Clima escolar; convivencia escolar; políticas de convivencia escolar; violencia escolar.

Abstract: School coexistence has been an object of research in areas ranging from school violence to educational inclusion. Being transformed into a topic that is not usually studied as the primary object, school coexistence is instead a variable associated with other factors, such as problems with learning outcomes, conflicts, bad teaching practices, inclusion, school violence, among others. For this reason raised the objective of analyzing the academic production published in Web of Science, Proquest, Scielo, and Scopus journals, in Latin America, from 2010 to June 2018, around the problems associated with school coexistence. For its development, it was decided to review the bibliography of a descriptive type, using 38 articles from different countries. One of the major results highlighted that the most researched topic is related to the factors (mentioned above) that influence school coexistence. Finally, it is worth mentioning that the association between school coexistence and school violence is one of the most outstanding characteristics.

Keywords: School atmosphere; school coexistence; school violence; policies of school coexistence. 
http://doi.org/10.15359/ree.24-2.17

ROR: https://ror.org/01t466c14 Universidad Nacional, Costa Rica

http://www.una.ac.cr/educare

educare@una.cr

Resumo: A convivência escolar tem sido investigada em áreas que vão desde a violência escolar até a inclusão educacional. Isso a transformou em um tópico que geralmente não é pesquisado como objeto principal. Mais bem, como uma variável associada a outros fatores, como: problemas com resultados de aprendizagem, conflitos, más práticas de ensino, inclusão, violência escolar, entre outros. Por isso, foi levantado o objetivo de analisar a produção acadêmica presente nas revistas Web ou Science, Proquest, Scielo e Scopus, da América Latina de 2010 a junho de 2018, em torno dos problemas associados à convivência escolar. Para o seu desenvolvimento decidiu-se rever a bibliografia de tipo descritivo, utilizando 38 artigos de diferentes países. Entre os principais resultados destaca-se que o tema mais pesquisado é aquele relacionado aos fatores (citados acima) que influenciam a convivência escolar. Por fim, vale ressaltar que uma das características mais marcantes é a associação entre a convivência escolar e a violência escolar.

Palavras chaves: Convivência escolar; clima escolar; políticas de convivência escolar; violência escolar.

\section{Introducción}

Los estudios sobre convivencia escolar (CE) han ido en aumento en los últimos años producto del alto nivel de violencia que sufren los niños y las niñas en las escuelas en América Latina. Estos niveles han alcanzado cifras preocupantes, como la mencionada por Soto y Trucco (2015) en el Capítulo III “Inclusión y contextos de violencia” del libro Juventud: Realidades y retos para un desarrollo con igualdad (Trucco y Ullmann, 2015). En dicho capítulo las autoras señalan que el $30 \%$ del estudiantado de América Latina y el Caribe afirma haber sido víctima de violencia escolar en cualquiera de sus dimensiones (por ejemplo físicas y psicológicas).

Junto a esta cifra se encuentran estudios realizados por UNESCO ${ }^{1}(2000,2010,2015)$, en los que se menciona que la violencia escolar es un factor recurrente que está afectando las instituciones educativas. Este fenómeno no solo incide en las aulas, sino, trasciende a la realidad social. No obstante, sería erróneo pensar que la sociedad replica lo que pasa en las escuelas. Más bien las escuelas son reproductoras de la sociedad (Bohórquez et ál., 2017; Chávez, 2017; Pacheco-Salazar, 2018; Trucco e Inostroza, 2017). Por lo tanto, si la violencia es un hecho común en las comunidades, entonces, niños, niñas y adolescentes resolverán los conflictos de forma negativa recurriendo a la agresión física o psicológica.

Sin duda, la preocupación por subsanar problemas relacionados con la violencia escolar a través de la CE es justificada. Por eso no es extraño encontrar estudios de CE que analizan temas como el bullying; discriminación; tipos, efectos o causas de la violencia; entre otros (Neut, 2017). Sin embargo, es importante enfatizar que la CE no es una subsección de la violencia escolar, simplemente se podría señalar como una "iniciativa" para promover en los niños, niñas, adolescentes y jóvenes el sano convivir (Trucco e Inostroza, 2017). Es decir, la violencia nace por malas prácticas de convivencia.

\footnotetext{
${ }^{1}$ Organización de las Naciones Unidas, para la Educación, la Ciencia y la Cultura (UNESCO, por sus siglas en inglés).
}

$$
2
$$

Jonathan Israel Andrades-Moya

Los artículos de la Revista Electrónica Educare del Centro de Investigación y Docencia en Educación de la Universidad Nacional, Costa Rica, se comparten bajo términos de la Licencia Creative Commons: Reconocimiento, № Comercial, Sin Obra Derivada 3.0 Costa Rica. Las autorizaciones adicionales a las aquí delimitadas se pueden obtener en el correo: educare@una.cr 
La CE es un concepto complejo de abordar y la mayoría de los estudios no explicitan una definición precisa. Generalmente se tiende a plantear una idea de cómo puede ser comprendida. Entre estas formas de comprender la CE se destacan las siguientes: 1) como la interrelación entre miembros de una comunidad (Ascorra et ál., 2016; García y Saavedra, 2016; Pulla, 2017; Viscardi, 2017); 2) como una forma de prevención de violencia escolar (Cuchan y D'Arcángelo, 2017; de Lellis y González, 2012); 3) como el conjunto de normas institucionales (Barreiro et ál., 2011; de Vasconcelos, 2017); 4) e incluso como una política educativa que debe ser trabajada en las instituciones (Bocanegra y Herrera, 2017). Todas ellas, políticas apuntadas a subsanar una serie de prácticas que afectan negativamente la CE de una institución educativa.

Dentro de estas malas prácticas se pueden encontrar algunos aspectos como: la exclusión, el bullying, la participación familiar, fallas en el ámbito normativo-prescriptivo de la institución escolar, comunicación o diálogo poco efectivo entre los diversos agentes escolares, entre otros (Chaparro et ál., 2015; Mendoza y Barrera, 2018).

En ese contexto, la CE se torna un eje de estudio necesario, ya que promueve el sano convivir en las comunidades educativas. Incluso en Latinoamérica se han promulgado diversas estrategias político/pedagógicas que apuntan, principalmente, al proceso de enseñanzaaprendizaje desde un plano formativo. Es decir, la CE se enseña, se aprende y se trabaja como un proceso construido por la comunidad (Ianni, 2003; lanni y Pérez, 2005). Desde esta óptica, y sumando la premisa de que la CE contribuye a disminuir la violencia escolar, este artículo se ha planteado como objetivo analizar la producción académica presente en revistas Web o Science, Proquest, Scielo y Scopus, de Latinoamérica, desde el 2010 hasta junio del 2018, en torno a las problemáticas asociadas a la convivencia escolar. A través de este objetivo se pretende responder a la siguiente pregunta: ¿Cuáles son los principales temas que se investigan sobre convivencia escolar en Latino América?

Por último, es necesario aclarar que este artículo no se guiará por una definición o forma de comprender la $C E$, ya que, se intenta ver la panorámica general y no solo aquellas que coincidan con una visión en particular.

\section{Metodología}

La investigación se basó en una revisión bibliográfica de tipo descriptiva, utilizando artículos científicos como fuentes primarias de información. La variable u objeto de estudio seleccionado fue la convivencia escolar (CE). La revisión bibliográfica se llevó a cabo en las bases de datos Web o Science, Proquest, Scielo y Scopus. La selección de estos fue a partir de 4 criterios: 1) artículos que incluyeran las palabras "convivencia escolar" o "convivencia en el contexto escolar" en el título, resumen o palabras claves; 2 ) artículos cuyos sujetos de estudio pertenecieran a países de América Latina; 3) artículos cuyo contexto de estudio perteneciera a países de América Latina; y 4) artículos publicados entre el año 2010 y junio del 2018. 
http://doi.org/10.15359/ree.24-2.17

ROR: https://ror.org/01t466c14 Universidad Nacional, Costa Rica

http://www.una.ac.cr/educare

educare@una.cr

En la búsqueda se obtuvieron aproximadamente 57 artículos. Posteriormente se realizó una lectura de cada uno de los artículos para comprobar la presencia de las palabras"convivencia escolar" o "convivencia en el contexto escolar" en sus títulos, resúmenes o palabras claves. A través de esta técnica se descartaron 5 artículos que no contenían dichas palabras en esas 3 secciones. Consiguientemente, se realizó una identificación de artículos duplicados, ya que, al recurrir a bases diferentes, se descargaron artículos alojados en dos o más de ellas. De esta acción se descartaron 14 archivos duplicados.

Según estos criterios fueron seleccionados 4 artículos de Argentina, 6 de Brasil, 12 de Chile, 7 de Colombia, 1 de Ecuador, 4 de México, 1 de República Dominicana y 3 de Uruguay, esto es, 38 artículos. Fueron organizados según su procedencia y procesados a través de una matriz de análisis (de construcción propia). En la matriz (ver Apéndice A) se contemplaron 3 categorías: 1) autorías del estudio y fecha; 2) temas de investigación; y 3) principales aportes (resultados o conclusiones) relacionados con la CE. Una vez completada la matriz con los 38 artículos, se procedió a clasificar tanto los temas de investigación, como las propuestas o aportes teórico/ empírico presentes en cada una.

La clasificación de los temas de investigación se desarrolló en la herramienta ofimática Excel. Para esto los temas fueron categorizados y posteriormente agrupados. Por ejemplo: el tema "análisis del clima social en relación con las normas perversas que pueden existir en los reglamentos de convivencia escolar" (Barreiro et ál., 2011), fue categorizado en "carácter normativo". Por lo tanto, cualquier tema relacionado directamente con las normas fue clasificado dentro de dicha categoría. En total se encontraron 10 categorías con 39 temas (un artículo tenía dos temas de investigación).

Las propuestas o aportes también fueron categorizados y agrupados y dieron un total de 68. Estos fueron organizados según la frecuencia con la que se repetían en los artículos. Para seleccionar las propuestas o aportes se consideraron todas las premisas y aseveraciones que contribuyeran al conocimiento teórico o a mejorar las prácticas sobre CE. Por ejemplo, D’Arcángelo (2016) señala que el estudiantado debe ser considerado un eje principal en una regulación escolar con base en la convivencia. Esta idea planteada por el autor fue considerada un aporte a la CE.

Con estas consideraciones, los resultados fueron organizados de dos formas: 1) un gráfico de barras que incluye la categorización de los temas; y 2) una tabla resumen que muestra la frecuencia de las propuestas o aportes teóricos/empíricos, realizados en los diversos artículos.

\section{Resultados}

Como se mencionó en la metodología, los resultados serán presentados en dos ejes. En primer lugar se dará a conocer el gráfico de barras que contiene la categorización de los temas y la cantidad de artículos que lo investigan. En segundo lugar se presenta una tabla resumen sobre las principales propuestas o aportes que se identificaron en los artículos. 


\section{Principales temas de investigación sobre convivencia escolar}

De los artículos seleccionados se han obtenido una serie de temas de investigación, los cuales fueron agrupados en 10 categorías. Cada una de ellas puede encontrarse en diferentes números de artículos (ver Figura 1).

Figura 1: Categorías obtenidas de los temas de investigación sobre convivencia escolar

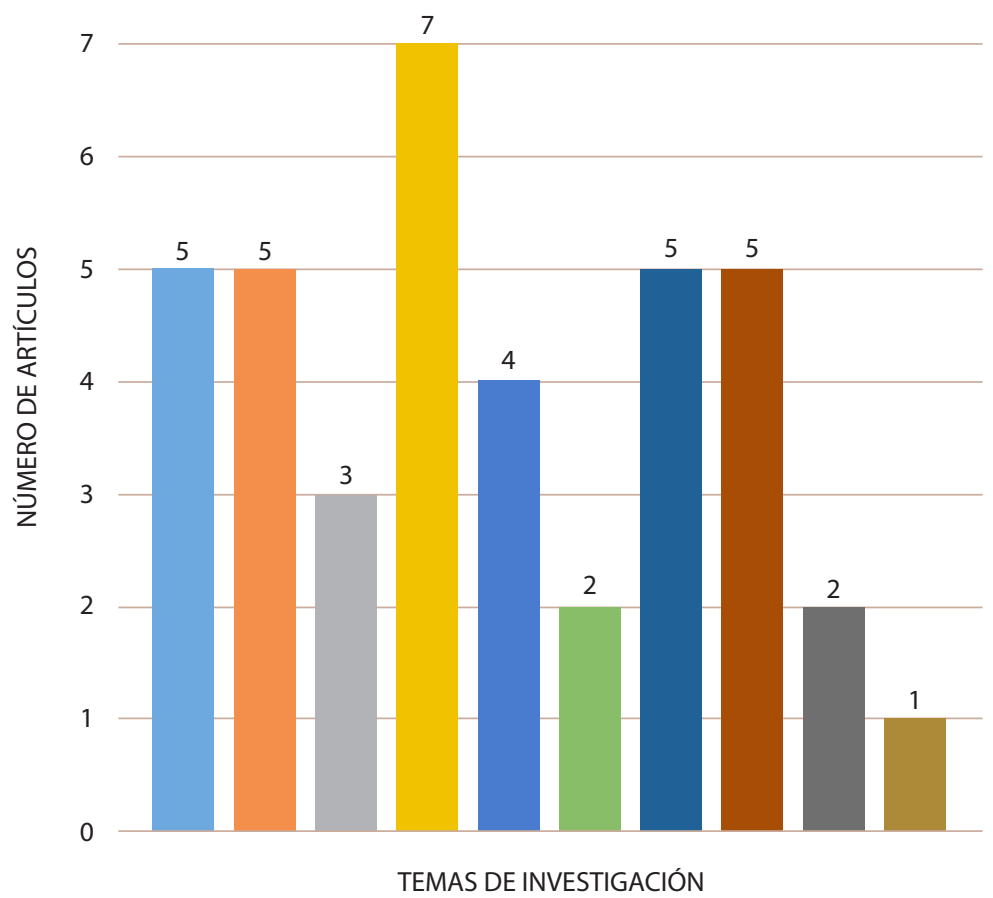

Formas de abordar la violencia escolar desde CE

Descripción de las políticas

Carácter normativo

Factores que influyen en la CE

Percepción sobre la CE

Instrumento de evaluación de la CE

Propuestas para mejorar la CE

Relación entre CE y otros factores

Descripción de agentes que regulan la CE

Comparación de prácticas de la CE

Nota: Elaboración propia.

La interpretación de resultados se realizará desde aquellas categorías que presentan menor cantidad de artículos hasta aquellas que presentan más. Es necesario especificar que se utiliza el concepto"cantidad"y no"relevancia", ya que no se intenta hacer un juicio de valor sobre la importancia de los temas. Simplemente presentarlos en un orden que permita mejor entendimiento.

En la Figura 1 se puede observar que existe 1 tema "comparación de prácticas de la CE" investigado exclusivamente en un artículo. Este artículo tiene como propósito comparar el estado de la CE en dos instituciones diferentes (Aristimuño y Noya, 2015). Una con alto índice de violencia escolar y otra que utiliza innovaciones educativas para mejorar la CE. En este estudio no se logra vislumbrar la comparación entre las prácticas educativas de los establecimientos. Solo se observa una descripción complementaria sobre dichas prácticas. 
http://doi.org/10.15359/ree.24-2.17

ROR: https://ror.org/01t466c14 Universidad Nacional, Costa Rica

http://www.una.ac.cr/educare

educare@una.cr

Prosiguiendo, en la Figura 1 se constatan dos temas que han sido investigados en dos artículos. El primero referente a "instrumentos de evaluación de la CE" y el segundo sobre la "descripción de agentes que regulan la CE".

Los artículos clasificados dentro de "instrumentos de evaluación de la CE", se enfocaban en medir diferentes factores de la CE. A saber: a) presentar y validar un cuestionario de CE que contemplara factores sobre la violencia (Muñoz et ál., 2017); y b) convivencia escolar democrática, inclusiva y pacífica (Chaparro et ál., 2015).

La segunda categoría, "descripción de agentes que regulan la CE", contaba con dos artículos que se preocupaban por describir las prácticas de dos tipos de agentes. En un artículo se hacía referencia a la persona encargada de CE (Valenzuela et ál., 2018); mientras que en otro a las personas fiscalizadoras estatales (Carrasco-Aguilar et ál., 2018). Estos fiscalizadores pueden ser agentes institucionales o agentes externos que evalúan el estado de la CE en las diferentes instituciones educativas. Ambos artículos coinciden en que ninguno de los dos roles tiene una función clara, lo que da lugar a desempeños no regulados y no orientados por políticas precisas. Esto provoca que sus derechos y deberes no sean cumplidos de forma adecuada.

En la Figura 1 también se evidencia 1 tema, "carácter normativo", que está presente en 3 artículos. Esta categoría hace alusión a la CE como un conjunto de normas. Por lo tanto los artículos correspondientes a esta categoría se preocupan por investigar los siguientes puntos: 1) análisis del clima social en relación con las normas perversas (Barreiro et ál., 2011); 2) reflexiones sobre indisciplina escolar, considerando las normas de CE (Boarini, 2013); y 3) conocer qué sabe el estudiantado sobre el reglamento escolar (Ochoa y Diez-Martínez, 2013).

Igualmente en la Figura 1 existe un tema estudiado en 4 artículos, "percepción sobre la CE". En este se encuentran investigaciones que describen las opiniones de ciertos actores educativos en relación con la CE en sus centros escolares. Respecto a esto: un artículo evalúa la percepción que se tiene de la CE en una institución inclusiva (Muñoz et al, 2014); otro da a conocer lo que piensa el profesorado acerca de su rol en la CE (Retuert y Castro, 2017); un tercer artículo identifica las creencias y prácticas que tienen miembros de una comunidad educativa sobre el conflicto (Bohórquez et ál., 2017); y un último artículo da a conocer la percepción que tienen los padres y madres sobre la gestión de la CE (Mendoza y Barrera, 2018).

Asimismo en la Figura 1 se puede observar que cuatro temas son investigados en 5 artículos cada uno. Estos temas son: "formas de abordar la violencia escolar desde CE", "propuestas para mejorar la CE", "descripción de las políticas" y "relación entre CE y otros factores".

En cuanto a la categoría "formas de abordar la violencia escolar desde CE", los estudios plantean la CE desde 3 visiones: 1) CE como regulación de la violencia escolar (Lopes y Gomes, 2012; de Vasconcelos, 2017); 2) CE como una forma de describir las percepciones y formas de prevenir la violencia escolar (de Lellis y González, 2012; Neut, 2017); o 3) CE como un mecanismo

6

Jonathan Israel Andrades-Moya

Los artículos de la Revista Electrónica Educare del Centro de Investigación y Docencia en Educación de la Universidad Nacional, Costa Rica, se comparten bajo términos de la Licencia Creative Commons: Reconocimiento, № Comercial, Sin 0bra Derivada 3.0 Costa Rica. Las autorizaciones adicionales a las aquí delimitadas se pueden obtener en el correo: educare@una.cr 
para conocer las concepciones de estudiantes y docentes sobre las causas de la violencia escolar (Pacheco-Salazar, 2018).

Respecto a la categoría "propuestas para mejorar la CE", los artículos tienen como propósito dar a conocer los resultados obtenidos al utilizar ciertas estrategias que contribuyan a mejorar la CE. En estos se pueden identificar: 1) capacitación sobre competencias sociales para el mejoramiento de la CE libre de violencia; 2 ) estrategia de construcción de normas de $C E$, de forma conjunta, entre la totalidad de miembros de una comunidad educativa (Nail et ál., 2018; Ossa et ál., 2016); 3) competencias psicosociales para mejorar la CE (Baquedano y Echeverría, 2013); y 4) uso de estrategias tecnológicas, ya sea la herramienta CLEHES (García y Saavedra, 2016) o las TIC (Rodríguez et ál., 2017).

En relación a la categoría"descripción de las políticas", se encontraron artículos que describen tanto políticas exclusivas de $\mathrm{CE}$, como políticas de educación en general. A saber: dos artículos analizan políticas argentinas sobre educación y CE (Cuchan y D’Arcángelo, 2017; D’Arcángelo, 2016); un artículo hace una reflexión sobre las políticas brasileñas (Eyng et ál., 2013); y dos artículos reflexionan sobre las políticas educativas colombianas (Bocanegra y Herrera, 2017; Orobio, 2014).

En cuanto a la categoría "relación entre CE y otros factores", agrupó a todos los factores que influyeran en la CE y que no habían sido categorizados anteriormente. Entre estos se encontraron los siguientes: a) juicio moral (de Oliveira et ál., 2010); b) clima escolar (Sandoval, 2014); c) rendimiento en matemática (Cerda et ál., 2017); d) segregación (Ascorra et ál., 2016); y e) la inteligencia emocional (Camacho et ál., 2017).

Estas investigaciones establecieron diferentes relaciones entre la CE y los factores nombrados. Por una parte los factores $a$, b y e presentan una relación directa con la CE. Es decir, mientras que la CE aumenta o disminuye, $a$, b y e, también aumentan o disminuyen. Por otra parte los factores c y d, presentan una relación inversa con la CE.

Por último, el tema más investigado sobre la CE es "factores que influyen en la CE", que agrupa 7 artículos. Estos artículos dan a conocer 6 factores que inciden, positiva o negativamente, en la CE. Estos son: 1) la pérdida de la memoria colectiva y la centralización de los estudios que descontextualizan los conocimientos (Fischmann, 2013); 2) las políticas públicas y la globalización (Orobio, 2014); 3) violencia escolar (Baridon y Martín, 2014; Malagón et ál., 2016); 4) las estrategias pedagógicas (Guerrero y Cepeda, 2016); 5) la práctica de valores (Pulla, 2017); y 6) prácticas y marcos políticos (Viscardi, 2017).

En síntesis, se puede indicar que los temas más investigados en relación a CE son: 1) factores que inciden en la $\mathrm{CE}$; 2 ) políticas educativas que contemplan la $\mathrm{CE}$; y 3) la relación entre CE y diversas variables. En cuanto a los temas menos investigados, se identificaron los siguientes: 1) comparación de prácticas sobre CE entre establecimientos; y 2) validación de instrumentos de medición de la CE. 


\section{Principales aportes sobre la convivencia escolar}

En cada uno de los artículos se detectó una gran cantidad de resultados, conclusiones o aportes hacia la educación. Además se identificaron una serie de propuestas sobre la CE. Estos fueron categorizados y clasificados por frecuencia de aparición. Es decir, por cada categoría obtenida, se especifica el número de artículos en los cuales aparece (ver Tabla 1).

En la Tabla 1 se puede apreciar que existen diversos aportes teóricos/empíricos sobre CE. Algunos de ellos fueron mencionados en un artículo, mientras que otros fueron señalados en ocho artículos.

En las categorías con mayor frecuencia se encontró una gran cantidad de artículos que plantean la necesidad de un cambio de perspectiva en los centros educativos. Estos sugieren que exista una transición desde la perspectiva disciplinar, hacia la perspectiva enfocada en la CE.

Otra categoría con alta frecuencia se refiere a la necesidad de mejorar la preparación de profesionales de la educación, para afrontar las exigencias que conlleva la CE. Estos artículos también indican que actualmente los grupos docentes no se encuentran preparados para asumir los nuevos desafíos socio-escolares, como la inclusión.

Las categorías señaladas en la Tabla 1 permiten visualizar dos temas interesantes de contemplar. Por una parte pueden contribuir a iniciar una perspectiva teórica sobre CE; por otra parte a identificar los temas menos explorados, tal como la preparación de profesionales de la educación en materias de CE.

Un tercer punto a mencionar hace referencia a las normas de CE. En los artículos se detectaron diversas ideas sobre las normas que pueden ser resumidas en 3 puntos: 1) las normas deben ser construidas de forma conjunta entre la totalidad de miembros de una institución educativa; 2 ) las normas deben ser claras, bien diseñadas, contextualizadas y consistentes; y 3) las respectivas sanciones aplicadas a quienes transgredan las normas deben ser de carácter formativo, dejando de lado el modelo punitivo.

Por último, en 5 artículos se indica que es necesario plantear propuestas para mejorar la CE. Esto es coincidente con lo señalado por lanni (2003) e lanni y Pérez (2005) quienes mencionan que la CE podría ser trabajada como un sistema que apunte a mejorar situaciones problemáticas. Es decir, la CE podría ser abordada como un proceso construido por quienes conforman una institución educativa. Todo, desde parámetros formativos y considerándola como un proceso de enseñanza/aprendizaje. Por ese motivo sería relevante asumir la CE como un eje de estudio principal y no solo como un componente que ayuda a describir relaciones o evaluar la violencia. 
Tabla 1: Frecuencia de las propuestas o aportes realizados en los artículos

\section{Categorías}

Se debe hacer un cambio de perspectiva desde la disciplinar hacia convivencia escolar

Falta de preparación profesional ante la convivencia escolar

Las normas deben ser construidas de forma colectiva

Aplicación inconsistente, ineficiente o desajustada de las políticas

El docente y la docente son fundamentales para privilegiar la convivencia escolar

Se deben plantear propuestas para mejorar la convivencia escolar

Cambiar de sanciones punitivas a formativas o valóricas

Las normas de convivencia inconsistentes o ineficientes, influyen negativamente en la CE

Los agentes reguladores de la CE no tienen un rol claro

El estudiantado debe ser visto como eje central de la convivencia escolar

El apoyo en las herramientas tecnológicas contribuye a mejorar la CE

El clima y convivencia escolar favorecen el aprendizaje

Existe una relación positiva entre inteligencia emocional y convivencia escolar

La CE no puede reducirse solo a las relaciones interpersonales.

La escuela es indispensable para enseñar a convivir

La violencia escolar es un factor descriptor de la CE

La centralización de las investigaciones descontextualiza los conocimientos sobre CE

La segregación afecta negativamente la convivencia escolar

Debe existir una intervención oportuna ante los conflictos

Existe una gran importancia en las normas para la regulación de la violencia escolar

Se entiende convivencia escolar más como la resolución de conflicto y no como la práctica de valores

Conceptualizar la CE es complejo, producto de las diversas perspectivas

Los padres y madres de familia tienen mayor compromiso según su nivel de satisfacción con el colegio
Número de artículos

Nota: Elaboración propia. 
http://doi.org/10.15359/ree.24-2.17

ROR: https://ror.org/01t466c14 Universidad Nacional, Costa Rica

http://www.una.ac.cr/educare

educare@una.cr

\section{Discusión}

En la sección anterior se pudo apreciar una serie de resultados que causan inquietud en relación con las investigaciones y a la CE como objeto de estudio. En primer lugar, es extraño contar con una cantidad baja de artículos. Esto si se considera que la CE es un tema que ha originado la promulgación de una serie de políticas y legislaciones a nivel latinoamericano. Además, es un tema frecuente en los medios de comunicación, que exponen continuamente episodios de bullying, violencia escolar, exclusión, fallas en los canales de comunicación entre familias y colegios, falta de liderazgo directivo, estrés en la labor docente, entre otros.

Este fenómeno puede producirse debido a que la CE se estudia de forma secundaria y muchas veces camuflada como un subtema de la violencia escolar. Es decir, la CE suele comprenderse como una forma de prevenir la violencia escolar (Carrasco-Aguilar et ál., 2018; Muñoz et ál., 2017; Sandoval, 2014). Por lo tanto, no sería extraño que en artículos de violencia escolar se desarrollen propuestas o descripciones que corresponden a la CE.

Como se observa, la CE es bastante asociada a la prevención de la violencia escolar. En efecto, sería de gran utilidad aumentar el número de investigaciones sobre cómo inciden los factores, ya mencionados, en la CE. De esta forma se podría contribuir a decrecer los altos niveles de agresión que se viven en Latinoamérica (Soto y Truco, 2015).

Otra posible explicación es la reducción del campo de estudio de la CE. En otras palabras, se han identificado una serie de estudios que abordan la CE como una forma de describir las interrelaciones entre miembros de una misma comunidad educativa (Cerda et ál., 2017; Muñoz, et ál., 2014; Retuert y Castro, 2017). Por lo tanto, al ser concebida solo como un descriptor general, podría obviarse la realización de investigaciones que midan la CE o que realicen propuestas. Esto también explicaría por qué estos temas son escasamente explorados.

Existe otra posible explicación enfocada en la necesidad de un cambio de perspectiva, desde el modelo disciplinario hacia la $C E$, que reportan una serie de investigaciones (por ejemplo: Cuchan y D’Arcángelo, 2017; de Lellis y González, 2012; Lopes y Gomes, 2012; de Vasconcelos, 2017). Estas investigaciones instan a proseguir con estudios sobre la CE. De esta forma se puede obtener información valiosa de cómo enfrentar, prevenir, abordar o trabajar las diferentes situaciones problemáticas que se viven en las escuelas.

Lo anterior provoca el siguiente planteamiento: si existen políticas y estudios internacionales sobre la importancia de la CE, ¿por qué aún sigue predominando el modelo disciplinario? ¿Será por desconocimiento y falta de preparación o por otros factores aún no identificados? Quizás esa sea la razón por la que se menciona que las políticas son poco efectivas. Es probable que los centros educativos no las estén aplicando como corresponde. También es probable que los estamentos encargados de formular estas políticas las diseñen de forma descontextualizada.Incluso, es posibleque estos no incluyan, dentro de sus actividades, la capacitación adecuada a los sujetos correspondientes.

10

Jonathan Israel Andrades-Moya

Los artículos de la Revista Electrónica Educare del Centro de Investigación y Docencia en Educación de la Universidad Nacional, Costa Rica, se comparten bajo términos de la Licencia Creative Commons: Reconocimiento, № Comercial, Sin 0bra Derivada 3.0 Costa Rica. Las autorizaciones adicionales a las aquí delimitadas se pueden obtener en el correo: educare@una.cr 
Respecto a los estudios latinoamericanos, hay que agregar que existen diferentes frecuencias de publicación de artículos sobre CE. Por una parte, la mayor cantidad de investigaciones son provenientes de Chile con 12 artículos. En segundo lugar, se encuentra Colombia con 7 artículos y en tercer lugar Brasil, con 6 artículos. Estos países concentran el 76 $\%$ de artículos en CE. Por otra parte, en países como Bolivia, Perú, Venezuela y Paraguay, no se detectaron investigaciones sobre esta materia.

Otro punto a mencionar es que menos del $15 \%$ de los artículos utilizaba algún instrumento que midiera la CE. La mayoría realizaba descripciones generales y poco profundas de las ópticas que tienen miembros de una comunidad escolar sobre la CE o de las políticas educativas.

También hay que indicar que dentro de los estudios solo 5 realizaban propuestas para mejorar la CE. En efecto, un 13 \% aborda la CE como un proceso construido de forma conjunta (lanni, 2003; lanni y Pérez, 2005). Con esto no se quiere decir que las investigaciones restantes no tengan peso teórico, solo se desea argumentar que los estudios sobre CE están menos enfocados en la acción y más en su descripción. En consecuencia, es posible que la CE sea considerada más desde componentes reactivos, que preventivos y formativos.

Por último, es importante mencionar que un $18 \%$ de los artículos contribuye con la identificación de factores que afectan a la CE. A saber: 1) la pérdida de la memoria colectiva y la centralización de los estudios que descontextualizan los conocimientos sobre $C E ; 2$ ) las políticas públicas y la globalización; 3) violencia escolar; 4) las estrategias pedagógicas; 5) la práctica de valores; y 6) prácticas y marcos políticos.

Esto es una base teórica importante que puede ser usada por otras investigaciones para conocer de mejor forma cómo afectan estos factores en la CE. También puede ser utilizada para determinar qué factor tiene más incidencia. Incluso puede contribuir a investigar el efecto de otros posibles factores que no hayan sido investigados o identificados en este artículo.

\section{Conclusión}

En el transcurso de este artículo se han identificado una serie de características y factores asociados a la CE. Dentro de estos es necesario enfatizar la importancia de plantear propuestas que contribuyan a mejorar las interrelaciones entre miembros de una institución.

Otro punto a considerar es la falta de preparación de las personas profesionales de la educación que se reporta en diversos artículos de CE. En estos no solo se alude al personal docente, también a personal directivo, asistente u otros agentes que desempeñen roles administrativos. Si bien se hace mayor referencia al cuerpo docente, esto no significa que solo este grupo carezca de preparación. 
Retomando un punto esbozado anteriormente, se ha detectado que la CE se concibe más de una forma reactiva que preventiva. Es decir, la CE se trabaja después de que haya sucedido un conflicto o alguna situación problemática y no desde un plano formativo. De esta forma no se contribuye: a) con aprendizaje de interacciones socio-escolares; b) a incrementar los resultados de aprendizaje; y c) a garantizar relaciones armónicas que permitan un óptimo desarrollo sociopersonal de todas las personas quienes conforman un centro educativo.

Por último, se invita a proseguir estudios sobre CE tomando alguna de las siguientes inquietudes: 1) de qué forma los centros de formación (universidades o institución técnica) preparan a sus profesionales de la educación en materias de $\mathrm{CE} ; 2$ ) qué está afectando a la poca eficacia de las políticas educativas: ¿será que son descontextualizadas y poco claras o que los establecimientos educacionales no están aplicándolas de la forma que corresponde?; 3) cómo lograr que la CE sea comprendida más de una forma predictiva que reactiva; y 4) contribuir a los conocimientos sobre CE desde los factores que influyen en ella y qué propuestas pueden plantearse para aminorar el efecto de estos sobre la CE.

\section{Referencias}

Aristimuño, A. y Noya, J. C. (2015). La convivencia escolar y el fenómeno del bullying en la enseñanza secundaria de Uruguay. Un estudio de caso. Páginas de Educación, 8(2), 201 224. https://doi.org/10.22235/pe.v8i2.691

Ascorra, P., López, V., Núñez, C. G., Bilbao, M. Á., Gómez, G. y Morales, M. (2016). Relación entre segregación y convivencia escolar en escuela públicas chilenas con altos puntajes PISA 2009. Universitas Psychologica, 15(1), 65-78. https://doi.org/10.11144/Javeriana.upsy15-1.rsce

Baquedano, C. y Echeverría, R. (2013). Competencias psicosociales para la convivencia escolar libre de violencia: Experiencia en una primaria pública de Mérida, Yucatán, México. Psicoperspectivas, 12(1), 139-160. https://doi.org/10.5027/psicoperspectivas-Vol12-Issue1fulltext-210

Baridon, D. y Martín, G. (2014). Violencia escolar en estudiantes de educación media. Ciencias Psicológicas, 8(2), 173-183. http://www.scielo.edu.uy/scielo.php?script=sci arttext\&pid $=$ S1688-42212014000200007

Barreiro, A., Beramendi, M. y Zubieta, E. (2011). ¿Normas perversas en el ámbito educativo? Aportes de psicología social. Ciencia, docencia y tecnología, 22(42), 137-154. http:// www.redalyc.org/articulo.oa?id $=14518444005$

Boarini, M. L. (2013). Indisciplina escolar: uma construção colectiva. Psicologia Escolar e Educacional, 17(1), 123-131. https://doi.org/10.1590/S1413-85572013000100013

12

Jonathan Israel Andrades-Moya

Los artículos de la Revista Electrónica Educare del Centro de Investigación y Docencia en Educación de la Universidad Nacional, Costa Rica, se comparten bajo términos de la Licencia Creative Commons: Reconocimiento, № Comercial, Sin Obra Derivada 3.0 Costa Rica. Las autorizaciones adicionales a las aquí delimitadas se pueden obtener en el correo: educare@una.cr 
Bocanegra, H. y Herrera, C. (2017). La Ley 1620 de 2013 y la política pública educativa de convivencia escolar en Colombia: Entre la formalidad jurídica y la realidad social. Revista Republicana, 23, 185-214. https://doi.org/10.21017/Rev.Repub.2017.v23.a36

Bohórquez, R. I., Chaux, Y. N. y Vaca, M. P. (2017). El conflicto en la convivencia escolar: Creencias y prácticas de estudiantes, padres de familia y docentes de una institución educativa distrital. Actualidades Pedagógicas, 70, 29-49. https://doi.org/10.19052/ap.4087

Camacho, N. M., Ordoñez, J. C., Roncancio, M. H. y Vaca, P. (2017). Convivencia escolar y cotidianidad: Una mirada desde la inteligencia emocional. Revista Educación y Desarrollo Social, 11(1), 24-47. https://doi.org/10.18359/reds.2649

Carrasco-Aguilar, C., Ascorra, P., López V.y Álvarez, J. P. (2018). Tensiones normativas de los fiscalizadores de la Superintendencia de Educación en la(s) política(s) de convivencia escolar en Chile. Perfiles educativos, 40(159), 126-143. https://doi.org/10.22201/ iisue.24486167e.2018.159.58153

Cerda, G., Salazar, Y., Sáez, K., Pérez, C. y Casas, J. A. (2017). Impacto de la percepción de los estudiantes respecto de la convivencia escolar sobre su rendimiento académico en Matemáticas. Psychology, Society, \& Education, 9(1), 147-161. https://doi.org/10.25115/ psye.v9i1.470

Chaparro, A. A., Caso, J., Fierro, M. C. y Díaz, C. (2015). Desarrollo de un instrumento de evaluación basado en indicadores de convivencia escolar democrática, inclusiva y pacífica. Perfiles Educativos, 37(149), 20-41. https://doi.org/10.22201/iisue.24486167e.2015.149.53118

Chávez, M. L. (2017). La violencia escolar desde la perspectiva infantil en el altiplano mexicano. Revista Mexicana de Investigación Educativa, 22(74), 813-835. http://www.scielo.org.mx/ scielo.php?script=sci abstract\&pid=S1405-66662017000300813\&lng=es\&nrm=iso

Cuchan, N. Y. y D’Arcángelo, M. B. (2017). Discursos gubernamentales en la redefinición de los vínculos en la escuela secundaria. Revista de educación, 27, 289-310. http://www.redalyc. org/jatsRepo/3845/384551991013/html/index.html

D’Arcángelo, M. B. (2016). Reconfiguraciones en el cambio de siglo: Educar en y para la democracia. Nuevos sentidos de la formación ciudadana. Espacios en Blanco. Revista de Educación, 26, 295-307. http://espaciosenblanco.unicen.edu.ar/numero anterior.xhtml;js essionid=A37A7EBE56B094BCD99423EA7D9B0999

de Lellis, M. y González, M. (2012). Concepciones y prácticas sobre la violencia en el ámbito escolar. Anuario de investigaciones, 19, 123-130. http://www.redalyc.org:9081/articulo. oa?id=369139948012 
de Oliveira, G. M., Caminha, I. de O. y de Freitas, C. M. S. M. (2010). Relações de convivência e princípios de justiça: a educação moral na escola. Psicologia Escolar e Educacional, 14(2), 261-270. https://doi.org/10.1590/S1413-85572010000200008

de Vasconcelos, I. C. O. (2017). Aprender a conviver, sem violência: o que dá e ñão dá certo? avaliação e Políticas Públicas em Educação, 25(97), 897-917. https://doi.org/10.1590/s0104$\underline{40362017002501180}$

Eyng, A. M., Gisi, M. L., Ens, R. T. y Pacievitch, T. (2013). Diversidade e padronização nas políticas educacionais: configurações da convivência escolar. Ensaio: avaliação e Políticas Públicas em Educação, 21(81), 773-800. https://doi.org/10.1590/S0104-40362013000400007

Fischmann, R. (2013). Memória colectiva e insurgência contra o olvido: em favor da convivência na escola. Ensaio: avaliação e Políticas Públicas em Educação, 21(81), 801-820. https://doi. org/10.1590/S0104-40362013000400008

García, O. y Saavedra Ulloa, M. S. (2016). Una herramienta ontológica y enactiva para la educación en gestión de convivencia. Orientación y Sociedad, 16, 59-66. http://sedici.unlp.edu.ar/ handle/10915/61929

Guerrero, H. R. y Cepeda, M. L. (2016). Uso de estrategias pedagógicas para el fortalecimiento de la convivencia escolar de jóvenes vulnerables. Revista de Pedagogia, 37(101), 57-79. http:// www.redalyc.org/articulo.oa?id=65950543004

lanni, N. D. (2003). La convivencia escolar: Una tarea necesaria, posible y compleja. Monografías virtuales, 2, 1-11. https://www.oei.es/historico/valores2/monografias/monografia02/ reflexion02.htm

Ianni, N. D. y Pérez E. (2005). La convivencia en la escuela: Un hecho, una construcción. Hacia una modalidad diferente en el campo de la prevención. Buenos Aires: Paidós.

Lopes, R. B. y Gomes C. A. (2012). Paz na sala de aula é uma condição para o sucesso escolar: que revela a literatura? Ensaio: avaliação e Políticas Públicas em Educação, 20(75), 261-282. https://doi.org/10.1590/S0104-40362012000200003

Malagón, E. L., Mateus, M. E. y Gómez, S. E. (2016). La convivencia escolar, un camino hacia la cultura de la paz. Educación y Ciudad, 31, 43-58. https://dialnet.unirioja.es/servlet/ articulo?codigo $=5803815$

Mendoza, B. y Barrera, A. (2018). Gestión de la convivencia escolar en educación básica: Percepción de los padres. Redie Revista Electrónica de Investigación Educativa, 20(2), 93102. https://doi.org/10.24320/redie.2018.20.2.1729 
Muñoz, F., Becerra, S.y Riquelme, E. (2017). Elaboración y validación psicométrica del cuestionario de convivencia escolar para la no violencia (CENVI). Estudios pedagógicos, 43(3), 205-223. https://doi.org/10.4067/S0718-07052017000300012

Muñoz, M. T., Lucero, B. A., Cornejo, C. A., Muñoz, P. A. y Araya, N. E. (2014). Convivencia y clima escolar en una comunidad educativa inclusiva de la provincia de Talca, Chile. Redie Revista Electrónica de Investigación Educativa, 16(1), 16-32. https://redie.uabc.mx/redie/article/ view/497/941

Nail, O., Valdivia, J., Gajardo, J., Viejo, C., Salas, R. y Romero, G. (2018). Estudio de casos: Tensiones y desafíos en la elaboración de la normativa escolar en Chile. Educação e Pesquisa, 44, 1-22. https://doi.org/10.1590/s1678-4634201711167834

Neut, P. (2017). Las violencias escolares en el escenario educativo chileno. Análisis crítico del estado del arte. Calidad en la Educación, 46, 222-247. https://doi.org/10.4067/S0718$\underline{45652017000100222}$

Ochoa, A. y Diez-Martínez, E. (2013). El reglamento escolar como eje de análisis de la convivencia en la escuela. Ensaio: avaliação e Políticas Públicas em Educação, 21(81), 667-684. https:// doi.org/10.1590/S0104-40362013000400003

Orobio, A. (2014). A propósito de la sociedad para la que educamos y el deterioro de la convivencia en los colegios de Bogotá. Praxis Pedagógica, 14(15), 221-229. https://revistas. uniminuto.edu/index.php/praxis/article/view/1003/943

Ossa, C., Figueroa, I. y Rodríguez, F. (2016). La metacognición institucional como herramienta para la mejora de la gestión de la convivencia escolar. Actualidades Investigativas en Educación, 16(3), 1-18. https://doi.org/10.15517/aie.v16i3.25962

Pacheco-Salazar, B. (2018). Violencia escolar: La perspectiva de estudiantes y docentes. Redie Revista Electrónica de Investigación Educativa, 20(1), 112-121. https://doi.org/10.24320/ redie.2018.20.1.1523

Pulla, J. F. (2017). La práctica de valores y su incidencia en la convivencia escolar. Revista Dilemas Contemponáneos:Educación, Política y Valores,4(2), 1-19. https:// dilemascontemporaneoseducacionpoliticayvalores.com/ files/200003417a6964a790e/17-1-28\%20La\%20pr\%C3\%A1ctica\%20de\%20valores\%20y\%20su\%20 incidencia\%20en\%20la\%20convivencia\%20escolar.....pdf

Retuert, G. y Castro, P. J. (2017). Teorías subjetivas de profesores acerca de su rol en la construcción de la convivencia escolar. Polis, Revista Latinoamericana, 16(46), 321-345. https://doi. org/10.4067/S0718-65682017000100321 
Rodríguez, B. P., Martínez, R. D. y Medina, C. P. (2017). Uso de las tecnologías de la información y la comunicación (TIC) como recurso didáctico promotor de convivencia escolar. Revista Espacios, 38(30), 1-9. http://hdl.handle.net/11323/1845

Sandoval, M. (2014). Convivencia y clima escolar: Claves de la gestión del conocimiento. Última Década, 41, 153-178. https://doi.org/10.4067/S0718-22362014000200007

Soto, H. y Trucco, D. (2015). Inclusión y contextos de violencia (Cap. 3). En D. Trucco y H. Ullmann, H. (Eds.), Juventud: Realidades y retos para un desarrollo con igualdad (pp. 115-170). CEPAL. https://doi.org/10.18356/1d4fe2ef-es

Trucco, D. e Inostroza, P. (2017). Las violencias en el espacio escolar. CEPAL. https://www.cepal. org/es/publicaciones/41068-violencias-espacio-escolar

Trucco, D. y Ullmann, H. (Eds.). (2015). Juventud: Realidades y retos para un desarrollo con igualdad. CEPAL. https://www.cepal.org/es/publicaciones/38978-juventud-realidades-retos-undesarrollo-igualdad

UNESCO. (2000). Marco de acción de Dakar. Educación para todos: Cumplir con nuestros compromisos comunes. UNESCO. https://unesdoc.unesco.org/ark:/48223/pf0000121147 spa

UNESCO. (2010). Factores asociados al logro cognitivo de los estudiantes de América Latina y el Caribe. Autor. https://unesdoc.unesco.org/ark:/48223/pf0000186769

UNESCO. (2015). Informe de resultados terce tercer estudio regional comparativo y explicativo. Factores asociados. Autor. https://unesdoc.unesco.org/ark:/48223/pf0000243533

Valenzuela, J., Ahumada, I., Rubilar, A., López, V. y Urbina, C. (2018). El encargado de convivencia escolar en Chile: Hacia la comprensión de su identidad laboral. Revista de Psicología, 36(1), 189-216. https://doi.org/10.18800/psico.201801.007

Viscardi, N. (2017). Adolescencia y cultura política en cuestión:Vida cotidiana, derechos políticos y convivencia en los centros educativos. Revista de Ciencias Sociales, 30(41), 127-158. http:// www.scielo.edu.uy/scielo.php?script=sci abstract\&pid=S0797-55382017000200127\&lng $=\mathrm{es} \& \mathrm{nrm}=\mathrm{iso}$ 
http://doi.org/10.15359/ree.24-2.17

ROR: https://ror.org/01t466c14 Universidad Nacional, Costa Rica

Apéndice A: Matriz de Análisis con las principales propuestas y aportes identificadas en los artículos

\begin{tabular}{|c|c|c|}
\hline Autor & Tema de investigación & Propuestas o aportes sobre CE \\
\hline 1. de Lellis y González (2012) & $\begin{array}{l}\text { Perspectiva de la violencia escolar, } \\
\text { desde la óptica de los implicados, } \\
\text { conociendo qué tipo de paradigma } \\
\text { es utilizado (el disciplinario o el de } \\
\text { convivencia). } \\
\text { "Convivencia como resolución de } \\
\text { conflicto o violencia escolar" } \\
\text { Eje iniciativo/político }\end{array}$ & $\begin{array}{l}\text { Las concepciones y prácticas sobre la violencia escolar son } \\
\text { tratadas (en mayor nivel) en el paradigma disciplinario. } \\
\text { Contribuye a su perpetuación. } \\
\text { Las iniciativas y propuestas que sugieren los actores están } \\
\text { en el paradigma de la convivencia. } \\
\text { La respuesta común y tradicional a la violencia escolar es } \\
\text { desde el paradigma disciplinar, mientras que las iniciativas } \\
\text { formuladas en los grupos focales se orientan hacia el para- } \\
\text { digma de la convivencia. Las personas autoras creen que es } \\
\text { por agotamiento del paradigma disciplinar, ya que no da } \\
\text { respuesta a lo requerido. }\end{array}$ \\
\hline
\end{tabular}

2. Cuchan y D'Arcángelo (2017) Recorrido en programas gubernamentales sobre política educativa.

"Convivencia como prevención de la violencia y formación valórica" Eje normativo/político

El discurso gubernamental argentino ha cambiado desde una perspectiva tradicional y disciplinaria de la educación y abordaje de la violencia escolar, hacia un enfoque en la convivencia.

3. Barreiro et ál. (2011)

Análisis del clima social en relación con las normas perversas que pudiesen existir en los reglamentos de convivencia escolar.

Denotan una importancia de las normas para la regulación social y que la presencia de normas perversas puede desencadenar la violencia escolar.

"Convivencia como construcción de reglamentos y/ normas" Eje institucional/regulativo

4. D’Arcángelo (2016)

6. Eyng et ál. (2013)
Análisis de la Ley Nacional n. ${ }^{\circ}$ 138/12 "Para la Promoción de la Convivencia y el Abordaje de la Conflictividad en las Instituciones Educativas"y reflexión sobre la postura del gobierno en la formación ciudadana y la incidencia de esta en las prácticas escolares.

"Convivencia como prevención de situaciones conflictivas" Eje normativo/político

5. de Vasconcelos (2017)

Presentar recomendaciones para eliminar o amenizar la violencia escolar y promover una adecuada convivencia. "Convivencia como normas" Eje normativo/político

Hace una reflexión sobre las políticas educacionales brasileñas y su relación con la convivencia escolar, específicamente en relación a lo que se explicita en el currículo y lo que se solicita en la educación, convivencia y estandarización respectivamente. "Convivencia como interacción entre los actores educativos"

Eje conceptual/definitorio
El autor señala que las estrategias que no funcionan son todas aquellas que llevan a sanciones físicas o punitivas, mientras que las estrategias enfocadas en temas valóricos, formativos, de diálogo, morales, éticos y ciudadanos (de interacción social).

Existe un discurso del profesorado enfocado a la falta de preparación y concientización ante la diversidad, lo que provoca una desaplicación de las políticas educativas y un refuerzo en la evaluación estandarizada.

Para lograr un espacio para la convivencia se debe dejar de lado el enfoque disciplinario.

La regulación escolar con base en la convivencia apunta a la participación del alumnado, no como eje secundario, más bien como eje central. Sobre todo en la restitución de sus derechos. 
http://doi.org/10.15359/ree.24-2.17

ROR: https://ror.org/01t466c14 Universidad Nacional, Costa Rica

http://www.una.ac.cr/educare

educare@una.cr

\begin{tabular}{|c|c|c|}
\hline Autor & Tema de investigación & Propuestas o aportes sobre CE \\
\hline 7. Boarini (2013) & $\begin{array}{l}\text { Presenta reflexiones sobre la indisciplina } \\
\text { escolar. Considera que las normas } \\
\text { de convivencia escolar son una } \\
\text { construcción colectiva. } \\
\text { "Convivencia como normas que regulan } \\
\text { la disciplina" } \\
\text { Eje normativo }\end{array}$ & $\begin{array}{l}\text { La indisciplina como acto humano natural; su reiteración } \\
\text { o incremento se debe a la calidad de la enseñanza, entre } \\
\text { otros. }\end{array}$ \\
\hline 8. Fischmann (2013) & $\begin{array}{l}\text { Hace una reflexión de cómo el olvido de } \\
\text { la memoria colectiva brasileña puede } \\
\text { afectar a la convivencia escolar. } \\
\text { "Convivencia como interacción } \\
\text { socioeducativa" } \\
\text { Eje conceptual/definitorio }\end{array}$ & $\begin{array}{l}\text { Se hace referencia a la pérdida de la memoria colectiva } \\
\text { la que se puede ver en muchos ámbitos, por ejemplo: en } \\
\text { escuelas ubicadas a la periferia en las que existe bilingüismo } \\
\text { o multilingüismo y todos se adaptan o se enseñan las diversas } \\
\text { lenguas para mejorar la convivencia. Esta pérdida, se hacenotar } \\
\text { en que no existen muchas investigaciones en estas escuelas } \\
\text { ya que todo está enfocado más bien en las metrópolis. }\end{array}$ \\
\hline
\end{tabular}

9. Lopes y Gomes (2012) Reflexiona acerca de los temas de la violencia escolar y da consejos o sugerencias de cómo abordarlos a través de las vías de la paz y del cambio de enfoque, pasando de uno autoritario a otro democrático y participativo.

"Convivencia como normas que regulan la disciplina"

Eje normativo

10. de Oliveira et ál. (2010)

Realiza una revisión bibliográfica acerca de la construcción del juicio moral y su relación con el saber convivir, la apropiación de valores y la comprensión de justicia.

"Convivencia como interacción entre los actores educativos y como proceso democrático" Eje conceptual

11. Muñoz et ál. (2014)
Evalúa la percepción de la convivencia y clima escolar en una institución inclusiva. "Convivencia como una construcción conjunta entre los actores educativos y como la interrelación entre estos" Eje normativo/conceptual
Realiza una argumentación teórica acerca de la relación entre convivencia y clima escolar con la gestión del conocimiento por parte de estudiantes. "Convivencia escolar como interrelación entre los actores educativos y como prevención de la violencia" Eje conceptual/preventivo
Se mencionan algunos procedimientos inadecuados que pueden promover la indisciplina: por una parte menciona prácticas autoritarias; por otra parte indica las normas poco claras, desajustadas, fallas de comunicación; y por último carencias profesionales en los educadores.

La moralidad está asociada al conjunto de normas y reglas que se han establecido. Además mencionan que la escuela debe ser vista como un espacio indispensable para la construcción de un mundo más igualitario y tolerante, que contribuye a la convivencia.

Se indica que es clave la preparación de docentes en temas de convivencia escolar y también ofrecer oportunidades de capacitación a los apoderados en este tema.

Otro punto importante es el de clarificar o agregar normas contextualizadas, además de construirlas en conjunto. Por último señalan que es relevante la intervención oportuna frente a situaciones de conflicto.

El generar un buen clima escolar incide en una convivencia escolar positiva por lo que esto contribuye al aprendizaje y los resultados. 
http://doi.org/10.15359/ree.24-2.17

ROR: https://ror.org/01t466c14 Universidad Nacional, Costa Rica

\begin{tabular}{ccc}
\hline Autor & Tema de investigación & Propuestas o aportes sobre CE \\
\hline
\end{tabular}

13. Valenzuela et ál. (2018)

14. Muñoz et ál. (2017)
Analizar el rol que tiene el encargado de la Ley n. ${ }^{\circ} 20.536$ sobre Violencia Escolar. "Convivencia como interrelación entre los actores educativos" Eje conceptual/político

Presentar y validar un cuestionario de "Convivencia como prevención de la violencia y como la interrelación entre los actores educativos" Eje normativo/conceptual

15. Nail et ál. (2018)

Da a conocer los resultados obtenidos convivencia escolar desde la propuesta de convivencia escolar para la no violencia. en una intervención socioeducativa con el fin de implementar un marco normativo construido por todos los actores de una comunidad educativa. "Iniciativa de CE. Construcción conjunta del marco normativo" Eje Propuesta

16. Cerda et ál. (2017)

17. Ossa et ál. (2016)

Da a conocer si existe una relación entre la percepción que tiene el estudiantado sobre su rendimiento en matemática. "Relación entre CE y desempeño académico (matemática)" Eje descriptivo/relacional

Analiza el concepto de metacognición institucional y su uso en la gestión de la convivencia escolar.

"Mejora de la convivencia" Eje Propuesta
No existe una claridad en el rol del encargado de convivencia escolar (ECE). Por una parte se tiende a confundir la identidad del ECE con la del inspector general; y por otra parte el ECE debe preocuparse por temas administrativos y de convivencia al mismo tiempo.

Indican que incorporar factores de violencia escolar a un cuestionario de convivencia permite conocer la gestión de CE que se realiza y así determinar si existe un deterioro de la convivencia.

Diferentes miembros de la comunidad educativa hicieron comentarios positivos de la propuesta.

Docentes y estudiantes presentan similar valorización a temas relacionados con la violencia escolar, pero diferentes en tanto a la utilización de dispositivos electrónicos.

Existe una relación estrecha e importante entre la percepción de la CE y los resultados obtenidos en matemática.

Se encontró una correlación negativa entre el grado de victimización y el rendimiento en matemática.

El rol docente como gestor del clima escolar es relevante y necesario para mejorar los resultados académicos.

La metacognición institucional contribuye a la gestión de la $\mathrm{CE}$, como por ejemplo: plantear propuestas que mejoren la resolución de conflicto.

Sin embargo, se señala que existen problemas en su implementación, tales como: capacidad de metacognición, tiempos y espacios, y gestión directiva y liderazgo.

Las políticas apuntan hacia una sana convivencia, sin embargo, asemejan la CE y la reducen a la violencia entre pares. conocimientos que se tiene respecto de la violencia escolar, abordando políticas que intentar subsanar este tema.

"Convivencia como prevención de la violencia"

Eje normativo/preventivo

19. Ascorra et ál. (2016) Da a conocer la relación entre la segregación y la calidad de la convivencia escolar.

"Relación de variables"

Eje descriptivo/relacional
Se concluye que existe una relación inversa entre segregación y calidad de convivencia escolar (CCE). Es decir, entre más segregado sea el establecimiento, menor es su calidad en la CE.

También existe una relación entre la dependencia de las instituciones con la CCE. Los municipales son de menor CCE. 
http://doi.org/10.15359/ree.24-2.17

ROR: https://ror.org/01t466c14 Universidad Nacional, Costa Rica

http://www.una.ac.cr/educare

educare@una.cr

\begin{tabular}{|c|c|c|}
\hline Autor & Tema de investigación & Propuestas o aportes sobre CE \\
\hline 20. Carrasco-Aguilar et ál. (2018) & $\begin{array}{l}\text { Da a conocer la forma en que los } \\
\text { fiscalizadores de la Superintendencia de } \\
\text { Educación enfrentan las tensiones entre el } \\
\text { contexto político y el escenario nacional. } \\
\text { "Convivencia escolar como normativa o } \\
\text { programa a fiscalizar" } \\
\text { Eje descriptivo/normativo }\end{array}$ & $\begin{array}{l}\text { El concepto de convivencia ha ido cambiando a lo largo de } \\
\text { los años desde el punto de "convivir con otros sujetos" a la } \\
\text { prevención de violencias. }\end{array}$ \\
\hline
\end{tabular}

21. Retuert y Castro (2017) Da a conocer qué es lo que piensa el profesorado sobre su rol en la convivencia escolar.

"Perspectiva de docente sobre convivencia escolar"

Eje descriptivo

22. García y Saavedra (2016)

Da a conocer los resultados de la implementación de la herramienta CLEHES, en relación con la gestión de CE.

"Propuesta para mejorar la gestión de

la CE"

Eje propuesta

23. Orobio (2014)

Reflexiona sobre las diferentes políticas públicas colombianas y el efecto de la globalización en las temáticas de convivencia escolar.

"Factores que afectan la convivencia" Eje descriptivo/factorial

24. Camacho et ál. (2017) Explorar la relación entre inteligencia emocional y convivencia escolar. "Relación entre convivencia e inteligencia emocional" Eje relacional

25. Bohórquez et ál. (2017)
Identificar las creencias y prácticas de miembros de una comunidad educativa frente al conflicto.

"Concepciones sobre la convivencia" Eje descriptivo
El personal docente cree tener un rol flexible en la $\mathrm{CE}$, ya que, depende de las características de sus estudiantes. tienen un enfoque tradicional de CE.

El personal docente ve la CE como la interrelación entre sujetos.

El programa CLEHES permite a estudiantes cambiar su visión de convivencia.

El deterioro de la CE parece ser afectada por las reformas económicas producto de la globalización, lo que provocó cambios de políticas educativas.

Existe una estrecha relación entre la inteligencia emocional y la CE. Con el aprendizaje de los aspectos emocionales, se privilegia el desarrollo de valores que potencian la CE.

El personal docente debe ser el garante de una buena relación entre estudiantes.

Las características socioculturales influyen las creencias y prácticas frente al conflicto.

Generalmente se aborda el conflicto desde las sanciones y de la violencia verbal o física. Esto producto de la influencia del ambiente y de los medios de comunicación. Se recomienda abordar el conflicto desde la CE y la generación de proyectos que privilegien la resolución positiva del conflicto.

26. Malagón et ál. (2016) Identificar los factores de agresión y violencia que afectan la $\mathrm{CE}$, para realizar una propuesta que promueva a la cultura de la paz.

"Factores que influyen en la CE"

Eje descriptivo/relacional

El conflicto prima en las realidades de los centros educativos de Colombia y no se aborda de una forma óptima, producto de las malas prácticas de docentes y estudiantes.

Se hace hincapié en hacer una propuesta pedagógica que contribuya a abordar el conflicto de una forma positiva, desde las nociones de la cultura de la paz. 
http://doi.org/10.15359/ree.24-2.17

ROR: https://ror.org/01t466c14 Universidad Nacional, Costa Rica

\begin{tabular}{cl}
\hline \multicolumn{1}{c}{ Autor } & \multicolumn{1}{c}{ Tema de investigación } \\
\hline 27. Bocanegra y Herrera (2017) & $\begin{array}{l}\text { Análisis de políticas educativas } \\
\text { relacionadas con la convivencia escolar. } \\
\text { "Descripción de las políticas de CE" } \\
\text { Eje descriptivo/normativo }\end{array}$ \\
\hline
\end{tabular}

28. Guerrero y Cepeda (2016)
Evalúa el uso de estrategias pedagógicas para el fortalecimiento de la convivencia escolar.

"Factores que influyen en la convivencia escolar"

Eje descriptivo/relacional
El marco político de Colombia crea una ley (Ley 1620 del 2013) que contribuya a la resolución de conflictos y a que los establecimientos se preocupen de aplicar las normativas relacionadas con la convivencia escolar como gestión institucional.

Existe una falta de uso de estrategias pedagógicas adecuadas y un manual de CE carente de normas que contribuyan a la convivencia.

Poco interés por parte de docentes hacia la resolución de conflictos.

El gobierno colombiano ha promulgado leyes que contribuyen y orientan a los establecimientos a generar una CE adecuada.

29. Rodríguez et ál. (2017)

Da a conocer el efecto del uso estratégico de las TIC para fomentar una sana convivencia escolar. "Propuesta para mejorar la CE" Eje descriptivo/propuesta

El uso de las TIC privilegia el desarrollo de una sana convivencia escolar. Estudiantes interiorizaron muchas habilidades y contenidos abordados en el blog que se utilizó como estrategia. En este blog, existían videos, relatos situacionales, formas de abordar conflictos, el manual de $\mathrm{CE}$, entre otros.

30. Pulla (2017)

Da cuenta de la incidencia de la práctica de valores en la convivencia escolar. "Factores que influyen en la CE" Eje descriptivo/factorial/relaciona

La convivencia es deficiente producto de las normas de CE poco óptimas.

Existe un problema generacional en la práctica de valores.

La CE no se analiza desde la práctica de valores, solo de la resolución de conflictos.

La CE se basa meramente en el cumplimiento de normas.

31. Viscardi (2017)

Da a conocer los elementos que surgen en la vida cotidiana educativa, desde temáticas como el problema de los valores, del respeto, del conflicto y del sentido de pertenencia. Además aborda puntos relacionados con factores estructurales, organizativos y de las principales políticas educativas sobre CE. “Descripción de factores que inciden en la convivencia escolar desde las prácticas y marcos políticos" Eje descriptivo

32. Aristimuño y Noya (2015) Da a conocer las diferentes formas de convivencia que existen en dos instituciones educativas de Uruguay. "Describe cómo es la convivencia en los centros educativos" Eje descriptivo

La pérdida de valores influye en la aparición de la violencia escolar.

Cambiar la matriz disciplinaria y punitiva, por una contextualizada y democrática.

Las personas autoras recomienda preparar a docentes en temas de convivencia, generar actividades de integración, crear acuerdos de convivencia democráticos, integrar a las familias, entre otras prácticas que privilegien la sana convivencia.

Eje descriptivo

33. Baridón y Martín (2014)

Da a conocer resultados de un estudio sobre violencia escolar en centros educativos de Uruguay.

"Convivencia relacionada con la violencia"

Eje descriptivo/relacional

Docente como pieza fundamental para combatir la violen-

Docente como pieza fundamental para com
cia escolar y generar espacios de mejor CE. 
http://doi.org/10.15359/ree.24-2.17

ROR: https://ror.org/01t466c14 Universidad Nacional, Costa Rica

http://www.una.ac.cr/educare

educare@una.cr

\begin{tabular}{lll}
\hline Autor & Tema de investigación & Propuestas o aportes sobre CE \\
\hline
\end{tabular}

34. Baquedano y Echeverría (2013) Experiencia de intervención en

Por su parte, las profesoras, al reflexionar sobre su práctica competencias sociales para el docente, reconocieron priorizar el castigo como medida mejoramiento de la CE libre de violencia. disciplinaria, pero también pudieron hacer conciencia Propuestas para mejorar la CE acerca de la poca efectividad de dicha medida. Docentes eje importante en la regulación de la CE. Algunas profesoras evitan el reporte a los padres y madres, debido a que consideran que eso agrava la violencia.

35. Chaparro et ál. (2015)
Diseña y valida un instrumento para la medición de la convivencia escolar. Instrumento de evaluación de la CE
Además, elaborar un constructo teórico sobre la convivencia como base para su operacionalización en procesos de evaluación de gran escala ofrece dificultades. El cuestionario debe ser perfeccionado, en cuanto a la cantidad de preguntas, las que deben ser reducidas. La evaluación de la convivencia escolar no puede reducirse a su dimensión interpersonal, toda vez que remite a la gestión escolar en todos sus ámbitos: la organización del trabajo en el aula, la organización escolar y la participación comunitaria.

La convivencia no puede limitarse solo a la coexistencia, ya que implica y supone una interacción de normas y valores que regulan las relaciones.

Cuando estos vehículos se basan en la fuerza de la autoridad más que en el diálogo, es difícil que el alumnado comprenda la importancia de las normas para la regulación de la convivencia en la escuela.

La convivencia escolar implica un aprendizaje complejo en dos sentidos: por un lado en la convivencia se aprenden las formas de relacionarnos con los demás y, por otro, aprendemos y enseñamos formas de apreciar y comprender el mundo y sus relaciones.

Docentes vitales para la regulación de la CE. Importancia de las normas. Construcción colectiva.

Las familias satisfechas sí aceptan la reparación del daño cuando su hijo o hija hizo daño a otro compañero o compañera (77\%), en contraste con las insatisfechas (45 $\%)$, estos resultados reflejan que las escuelas comienzan a promover la participación de los padres y madres de familia en la mejora de la convivencia escolar. Identifica que quienes sienten satisfacción con la atención general que se brinda en la institución escolar participarán más. La efectividad en la gestión de los planteles escolares para mejorar la convivencia entre escolares implica hacer uso de la información brindada a través de encuestas, evaluaciones y diagnósticos, lo que facilitará, sin duda, la toma de decisiones y la implementación de acciones. 
http://doi.org/10.15359/ree.24-2.17

ROR: https://ror.org/01t466c14 Universidad Nacional, Costa Rica

http://www.una.ac.cr/educare educare@una.cr

\begin{tabular}{cll}
\hline \multicolumn{1}{c}{ Autor } & \multicolumn{1}{c}{ Tema de investigación } & \multicolumn{1}{c}{ Propuestas o aportes sobre CE } \\
\hline 38. Pacheco-Salazar (2018) & $\begin{array}{l}\text { Conoce las concepciones que tienen } \\
\text { estudiantes y docentes sobre las causas } \\
\text { de la violencia escolar. }\end{array}$ & $\begin{array}{l}\text { Las relaciones interpersonales son consideradas un } \\
\text { indicador de convivencia armónica y factor de protección } \\
\text { ante la violencia escolar }\end{array}$ \\
& $\begin{array}{ll}\text { Formas de abordar la violencia escolar } \\
\text { desde la CE }\end{array}$ & $\begin{array}{l}\text { En los centros educativos se recrean y construyen modelos } \\
\text { de convivencia social. Por tanto, es relevante que los } \\
\text { programas educativos destinados a prevenir y erradicar la } \\
\text { violencia escolar consideren el trabajo desde la educación } \\
\text { emocional, la atención a la diversidad, y el aprendizaje y el } \\
\text { trabajo cooperativos. }\end{array}$ \\
\hline
\end{tabular}

Nota: La matriz es de creación propia, pero la información pertenece a cada uno de las autoras y autores citados. 